\title{
Huiles et cires comme agents de démoulage dans I'industrie agro-alimentaire
}

\author{
Annie ROYER \\ Bernard PRILLEUX \\ SIO, Société Industrielle des Oléagineux, \\ Groupe ADM, \\ 16 rue du Général de Gaulle, \\ 62053 Saint Laurent Blangy Cedex \\ $<$ Annie.Royer@adm.com >
}

\begin{abstract}
Oils and waxes as release agents in food industry
To prevent adhesion in the mould, release agents are commonly used in food industry, such as bakery, pastry, meat processing industry, confectionery, or cheese industry. Over the past two decades, petroleum based products such as petroleum jelly and paraffin have been replaced by mixture of vegetable oils (soy, canola, etc.), plant waxes (candelilla wax, carnauba wax), emulsifiers (soy or rape seed lecithin, mono- or diglycerides) and sometimes antioxidants. Here is a short overview of their main characteristic.
\end{abstract}

Key words: release agents, vegetable oils, plant waxes, emulsifiers

ques telles que la bonne aptitude à la lubrification, la bonne aptitude à la pulvérisation, l'efficacité sur un grand intervalle de température, une bonne stabilité physique afin d'éviter les phénomènes de déphasage, de décantation.

Ils doivent également offrir un certain nombre de caractéristiques chimiques: bonne stabilité à l'oxydation, point de fumée élevé, faible tendance à la polymérisation afin d'éviter l'encrassement des moules.

Enfin, sur le plan organoleptique, les démoulants doivent avoir un goût et une odeur neutres afin de ne pas perturber la qualité du produit de base.

\section{Deux catégories de démoulants}

Deux catégories de démoulants cohabitent et répondent à l'ensemble des critères précités. Les premiers contiennent de l'eau et sont donc obtenus par une émulsion eau-huile. L'eau est ainsi emprisonnée dans des globules d'huile ou de graisse. Afin d'obtenir une émulsion bien stable, l'ajout d'émulsifiants s'avère souvent nécessaire.

Seconde catégorie : les démoulants qui ne contiennent pas d'eau et sont donc $100 \%$ anhydres. Ils sont à base d'huile végétale, peuvent contenir de la lécithine ou des antioxydants, et surtout des agents texturants tels que les mono- et diglycérides, cires végétales ou huiles totalement hydrogénées afin d'ajuster la viscosité du démoulant soit au type d'utilisation envisagée (rouleau, pinceau, pistolet, etc.), soit au type de surface envisagée (paroi verticale ou paroi horizontale).

Dans le cas d'une lubrification de moules avec parois verticales, il se révèle bien souvent nécessaire d'utiliser des démoulants ayant une viscosité relativement élevée. Le challenge à relever pour ce type de démoulant est l'obtention d'un mélange entre de l'huile et un ingrédient à haut point de fusion (cire, huile totalement hydrogénée, monoou diglycérides) qui reste à la fois homogène, stable physiquement pendant toute la durée du stockage; sans agglomérats ou particules qui viendraient boucher les buses de pulvérisation et entraîneraient une absence de

Ces mélanges doivent bien évidemment répondre à des caractéristiques techni-

Pour citer cet article : Royer A, Prilleux B. Huiles et cires comme agents de démoulage dans l'industrie agro-alimentaire. OCL 2012; 19(2) : 123-124. doi : 10.1684/ocl.2012.0436 
lubrification des moules sur la ligne de production.

\section{Exemples}

Nous présentons dans le tableau 1 quelques exemples de composition de démoulants et les domaines d'application recommandés.
Tableau 1. Exemples de composition de démoulants et les domaines d'application recommandés.

\begin{tabular}{|llll|}
\hline Démoulants & A & B & C \\
\hline $\begin{array}{l}\text { Domaines } \\
\text { d'application }\end{array}$ & $\begin{array}{l}\text { Boulangerie, biscuiterie, } \\
\text { biscotterie }\end{array}$ & $\begin{array}{l}\text { Boulangerie, industrie } \\
\text { fromagère }\end{array}$ & $\begin{array}{l}\text { Moules et plaques } \\
\text { en boulangerie }\end{array}$ \\
\hline Composition & $\begin{array}{l}\text { Huiles végétales, cire } \\
\text { végétale (canauba), } \\
\text { lécithine }\end{array}$ & $\begin{array}{l}\text { Huiles végétales, TCM, } \\
\text { lécithine, antioxydant }\end{array}$ & $\begin{array}{l}\text { Huiles végétales, } \\
\text { lécithine }\end{array}$ \\
\hline
\end{tabular}

